Bull. Soc. math. France

131 (2), 2003, p. 289-306

\title{
FOCUSING OF A PULSE WITH ARBITRARY PHASE SHIFT FOR A NONLINEAR WAVE EQUATION
}

\author{
By RÉmi CaRles \& David Lannes
}

\begin{abstract}
We consider a system of two linear conservative wave equations, with a nonlinear coupling, in space dimension three. Spherical pulse like initial data cause focusing at the origin in the limit of short wavelength. Because the equations are conservative, the caustic crossing is not trivial, and we analyze it for particular initial data. It turns out that the phase shift between the incoming wave (before the focus) and the outgoing wave (past the focus) behaves like $\ln \varepsilon$, where $\varepsilon$ stands for the wavelength.

RÉSUMÉ (Focalisation d'impulsion et déphasage arbitraire pour une équation des ondes non-linéaire)

Nous considérons un système de deux équations des ondes linéaires conservatives, couplées non-linéairement, en dimension trois d'espace. Pour des données initiales radiales de type impulsions courtes, les solutions focalisent à l'origine lorsque la longueur d'onde tend vers zéro. Le caractère conservatif de l'équation fait que la traversée de la caustique n'est pas triviale : nous l'analysons pour des données initiales particulières. Il ressort que le déphasage entre l'onde entrante (avant focalisation) et l'onde sortante (après focalisation) se comporte en $\ln \varepsilon$, où $\varepsilon$ représente la longueur d'onde.
\end{abstract}

Texte reçu le 14 mars 2002, révisé le 19 septembre 2002, accepté le 23 octobre 2002 RÉmi CARLES, MAB, UMR CNRS 5466, Université Bordeaux 1, 351 cours de la Libération, 33405 Talence Cedex (France) • E-mail : carles@math.u-bordeaux.fr DAVID LANNES, MAB, UMR CNRS 5466, Université Bordeaux 1, 351 cours de la Libération, 33405 Talence Cedex (France) • E-mail : lannes@math.u-bordeaux.fr 2000 Mathematics Subject Classification. - Primary 35B40. Secondary 35L70, 35P25. Key words and phrases. - Nonlinear geometric optics, short pulses, caustic, long range scattering. 


\section{Introduction}

1.1. Motivation. - Informations on rapidly oscillating solutions to partial differential equations can be provided by WKB approximations, whose first rigorous justification goes back to Lax [14] (see also [12] for a survey of more recent results). This approach yields good results as long as the solution of the eikonal equation remains smooth, that is, before caustics are formed. The influence of such a singular locus on the behavior of solutions to linear partial differential equations was explained by Ludwig [15], and Duistermaat [9]; the caustic crossing is mainly described by the Maslov index.

For nonlinear equations, no global theory is available. Formal computations on conservation laws performed in [10] suggest the existence of two distinct notions of critical indexes; a critical index to describe the solution away from caustics, and another one to analyze the solution near caustics. Rigorous proofs for results similar to those stated in [10] are given in [13], [2], and in the more recent articles by Carles and Rauch in the case of pulse-like data (as opposed to wave trains, see e.g. [1], [7]), as we now recall.

Consider the initial value problem,

$$
\left\{\begin{array}{l}
\left(\partial_{t}^{2}-\Delta\right) u^{\varepsilon}+a \varepsilon^{p-2}\left|\partial_{t} u^{\varepsilon}\right|^{p-1} \partial_{t} u^{\varepsilon}=0, \quad(t, x) \in[0, T] \times \mathbb{R}^{3}, \\
u_{\mid t=0}^{\varepsilon}=\varepsilon U_{0}\left(r, \frac{r-r_{0}}{\varepsilon}\right), \quad \partial_{t} u_{\mid t=0}^{\varepsilon}=U_{1}\left(r, \frac{r-r_{0}}{\varepsilon}\right),
\end{array}\right.
$$

where $p \geq 2, r=|x|$ and $r_{0}>0$. The parameter $\varepsilon$ lies in $\left.] 0,1\right]$, and we want to analyze the asymptotics of $\partial_{t} u^{\varepsilon}$ in $L^{\infty}$ as $\varepsilon$ goes to zero. We assume that the functions $U_{0}$ and $U_{1}$ are infinitely differentiable, bounded, and compactly supported in $r>0$. The last assumption implies that the initial data are pulse like in the limit $\varepsilon \rightarrow 0$. The spherical symmetry of the initial data causes focusing at the origin at time $t=r_{0}$.

The balance between the power of $\varepsilon\left(\varepsilon^{p-2}\right)$ and the power of the nonlinearity $\left(\left|\partial_{t} u^{\varepsilon}\right|^{p-1} \partial_{t} u^{\varepsilon}\right)$ corresponds to the critical notion of "nonlinear caustic", as named in [10]; this means that nonlinear effects occur at leading order near the focus $(t, x)=\left(r_{0}, 0\right)$, whereas it would not be so if $\varepsilon^{p-2}$ was replaced by $\varepsilon^{\delta}$ with $\delta>p-2$ (see [7] for the case $\delta=0,1<p<2$ ).

In [5], [6], the following distinctions were proved in the case $a>0$, that is when the equation is dissipative (see also [4], [8]).

- If $p>2$, the solution $u^{\varepsilon}$ passes through the focus, and the caustic crossing is described by a (short range) scattering operator, associated to Eq. (1.1) with $\varepsilon=1$ (see [6]).

- If $p=2$, then the pulses are absorbed before reaching the focus ([5], see also [11], [13]).

The case $p>2, a \in \mathbb{C}$ is also considered in [4], for small data $U_{j}$, with the same conclusion as in the case $a>0$. It is described by a scattering operator,

TOME $131-2003-\mathrm{N}^{\mathrm{O}} 2$ 
and the analysis suggests that the equivalent problem for $a>0, p=2$ leads to a long range scattering operator. The second point would therefore mean that for a dissipative equation, the image of a long range scattering operator may be reduced to the zero function. On the other hand if $a$ is a pure imaginary, then Eq. (1.1) is conservative, therefore the pulses are not absorbed, and the underlying long range scattering operator should not be trivial. We therefore consider in the present article the case where $a$ is a pure imaginary, and $p=2$.

In [3], the cubic nonlinear Schrödinger equation is analyzed is one space dimension. A semi-classical analysis shows that when suitable initial data are considered, then the solution focuses at one point, and the caustic crossing is described by a long range scattering operator, which gives rise to a "random" phase shift past the focus, inasmuch as it depends on $\varepsilon$ (logarithmically). The nonlinear Schrödinger equation which was considered is conservative, but one could argue that the geometry associated to this problem is not natural. This is why we consider here the wave equation, with the idea of underscoring the corresponding phenomenon of arbitrary phase shift (see Th. 1.1 below).

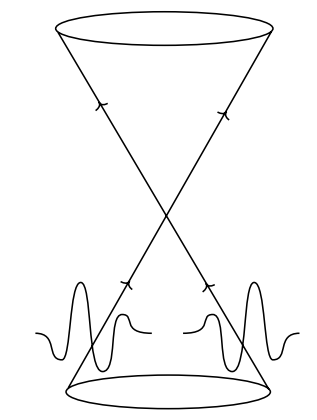

Figure 1. Focusing of pulses in the case of radially symmetric initial data for the wave equation.

1.2. Reduction of the problem. - It turns out that the initial value problem (1.1) with $a \in i \mathbb{R}$ and $p=2$ is technically quite difficult to analyze, with an asymptotic description of the solution in mind. We therefore consider the simplified problem,

$$
\left\{\begin{array}{l}
\left(\partial_{t}^{2}-\Delta\right) \mathbf{u}^{\varepsilon}=0, \\
\left(\partial_{t}^{2}-\Delta\right) u^{\varepsilon}-4 i\left|\partial_{t} \mathbf{u}^{\varepsilon}\right| \partial_{t} u^{\varepsilon}=0 .
\end{array}\right.
$$

This is a system of two linear equations, with a nonlinear coupling that corresponds to a semi-implicit scheme that preserves the conservation of the energy, in view of a numerical treatment for instance. We picked $a=-4 i$ for simpler notations in the sequel. 
We now proceed to the same reduction as in [7] and [4]. Since the initial data are spherical, so is the solution so, with the usual abuse of notation,

$$
u^{\varepsilon}(t, x)=u^{\varepsilon}(t,|x|), \quad u^{\varepsilon}(t,|x|) \in C_{\text {even in } r}^{\infty}\left(\mathbb{R}_{t} \times \mathbb{R}_{r}\right) .
$$

Introduce $v^{\varepsilon}:=\left(v_{-}^{\varepsilon}, v_{+}^{\varepsilon}\right)$ where

$$
\tilde{u}^{\varepsilon}(t, r):=r u^{\varepsilon}(t, r), \quad v_{\mp}^{\varepsilon}:=\left(\partial_{t} \pm \partial_{r}\right) \tilde{u}^{\varepsilon} .
$$

Then (1.2) becomes

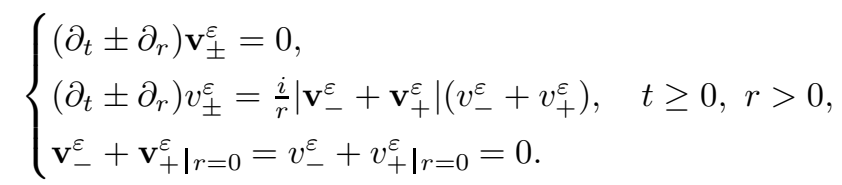

We now turn to the choice of the initial data. As shown in [7], the interaction of the outgoing wave $\left(v_{+}^{\varepsilon}\right)$ and the incoming wave $\left(v_{-}^{\varepsilon}\right)$ is negligible outside the focus, because of the pulse like aspect of the waves (they do not have time to interact), therefore we simplify the notations by imposing $\mathbf{v}_{+\mid t=0}^{\varepsilon}=v_{+\mid t=0}^{\varepsilon}=0$. We also choose

$$
\mathbf{v}_{-\mid t=0}^{\varepsilon}=f\left(\frac{r-r_{0}}{\varepsilon}\right),
$$

where $f \in C_{0}^{\infty}(\mathbb{R})$. We removed the dependence of the initial data upon slow variables, for it is negligible because of the pulse like aspect. We therefore have explicitly, for $t \geq 0$,

$$
\mathbf{v}_{-}^{\varepsilon}(t, r)=f\left(\frac{r+t-r_{0}}{\varepsilon}\right), \quad \mathbf{v}_{+}^{\varepsilon}(t, r)=-f\left(\frac{t-r-r_{0}}{\varepsilon}\right) .
$$

The expression of $\mathbf{v}_{+}^{\varepsilon}$ shows that on traversing the focus the amplitude of the profile is multiplied by $-1=\mathrm{e}^{i \mathbf{2 \pi / 2}}$. This phenomenon is linear: it is the classical Maslov index for a focal point of multiplicity equal to 2 (see e.g. [9]).

The choice of $v_{-\mid t=0}^{\varepsilon}$ may seem more intricate, but it turns out that it simplifies the computations (at least it makes them feasible) and leads to the phenomenon we want to underscore. We choose

$$
v_{-\mid t=0}^{\varepsilon}=g\left(\frac{r-r_{0}}{\varepsilon}\right) \mathrm{e}^{i\left|f\left(\left(r-r_{0}\right) / \varepsilon\right)\right| \ln r_{0} \varepsilon / r},
$$

with $g \in C_{0}^{\infty}(\mathbb{R})$. The introduction of a logarithmic factor in the phase may seem artificial, just as well as in [3]. Remind that our goal is to describe the caustic crossing thanks to a long range scattering operator: it is classical that this analysis involves phase modification. It will appear later on that our proofs highly rely on this particularity (see Remark 4.2), and it would be interesting to know what happens when this initial phase term is removed. On the other hand, the presence of $r_{0} / r$ in the logarithmic term is purely cosmetic, to simplify as much as possible the notations in the sequel. It could be removed, essentially because on the support of $f$, we have $r-r_{0}=O(\varepsilon)$.

TOME $131-2003-\mathrm{N}^{\mathrm{O}} 2$ 
The reduced problem we will study therefore reads,

$$
\left\{\begin{array}{l}
\left(\partial_{t} \pm \partial_{r}\right) v_{ \pm}^{\varepsilon}=\frac{i}{r}\left|f\left(\frac{r+t-r_{0}}{\varepsilon}\right)-f\left(\frac{t-r-r_{0}}{\varepsilon}\right)\right|\left(v_{-}^{\varepsilon}+v_{+}^{\varepsilon}\right) \\
v_{-}^{\varepsilon}+v_{+}^{\varepsilon} \mid r=0=0 \\
v_{-\mid t=0}^{\varepsilon}=g\left(\frac{r-r_{0}}{\varepsilon}\right) \mathrm{e}^{i\left|f\left(\left(r-r_{0}\right) / \varepsilon\right)\right| \ln r_{0} \varepsilon / r} \\
v_{+\mid t=0}^{\varepsilon}=0
\end{array}\right.
$$

1.3. Statement of the results. - In the rest of this paper, we analyze the reduced functions $v_{ \pm}^{\varepsilon}$. One could deduce the asymptotics of $\partial_{t} u^{\varepsilon}$ in $L^{\infty}$ thanks to (1.3). The main result of this article is the following.

TheOREM 1.1. - Let $f, g \in C_{0}^{\infty}(\mathbb{R}), r_{0}>0, \varepsilon>0$. Then (1.6) has a unique, global solution $\left(v_{-}^{\varepsilon}, v_{+}^{\varepsilon}\right) \in L^{\infty}\left(\mathbb{R}_{+} \times \mathbb{R}_{+}\right)^{2}$, uniformly bounded for $\left.\left.\varepsilon \in\right] 0,1\right]$. Moreover, one has the following asymptotics, as $\varepsilon$ goes to zero. Let $C>0$.

- If $0 \leq t \leq r_{0}-C \varepsilon$, then

$$
\begin{aligned}
\left\|v_{-}^{\varepsilon}(t, r)-g\left(\frac{r+t-r_{0}}{\varepsilon}\right) \mathrm{e}^{i\left|f\left(\left(r+t-r_{0}\right) / \varepsilon\right)\right| \ln r_{0} \varepsilon / r}\right\|_{L_{r}^{\infty}} & +\left\|v_{+}^{\varepsilon}(t, r)\right\|_{L_{r}^{\infty}} \\
& =O\left(\frac{\varepsilon}{r_{0}-t}\right) .
\end{aligned}
$$

- There exists $\nu_{+} \in L^{\infty}(\mathbb{R})$ such that for $t \geq r_{0}+C \varepsilon$,

$$
\begin{aligned}
\left\|v_{+}^{\varepsilon}(t, r)-\nu_{+}\left(\frac{t-r-r_{0}}{\varepsilon}\right) \mathrm{e}^{i \theta^{\varepsilon}(t, r)}\right\|_{L_{r}^{\infty}}+\left\|v_{-}^{\varepsilon}(t, r)\right\|_{L_{r}^{\infty}} & =O\left(\varepsilon+\frac{\varepsilon}{t-r_{0}}\right),
\end{aligned}
$$

where $\theta^{\varepsilon}$ is given by

$$
\theta^{\varepsilon}(t, r)=\int_{r_{0}}^{r / \varepsilon} \frac{1}{\sigma}\left|f\left(\frac{t-r-r_{0}}{\varepsilon}+2 \sigma\right)-f\left(\frac{t-r-r_{0}}{\varepsilon}\right)\right| \mathrm{d} \sigma .
$$

- There exists a "caustic profile" $\left(\mathrm{V}_{-}, \mathrm{V}_{+}\right) \in L^{\infty}\left(\mathbb{R} \times \mathbb{R}_{+}\right)^{2}$ such that for $\left|t-r_{0}\right| \leq C \varepsilon$ and $r \leq C \varepsilon$,

$$
v_{ \pm}^{\varepsilon}(t, r)=\mathrm{V}_{ \pm}\left(\frac{t-r_{0}}{\varepsilon}, \frac{r}{\varepsilon}\right)+O(\varepsilon) .
$$

REMARK 1.2. - The constant $C$ in the above statement is arbitrary, its influence is hidden in the remainders. Notice that when $t-r_{0}=O(\varepsilon)$, the first two assertions claim nothing more than the uniform boundedness of $v_{-}^{\varepsilon}$ and $v_{+}^{\varepsilon}$.

REMARK 1.3. - We will prove in Sect. 4 that $\nu_{+}$is not only bounded, but has algebraic decay, $\nu_{+}(\lambda)=O\left(\langle\lambda\rangle^{-1}\right)$, where as usual, $\langle\lambda\rangle=\sqrt{1+\lambda^{2}}$. 
REMARK 1.4. - The phase shift between the incoming and the outgoing waves reads

$$
\theta^{\varepsilon}(t, r)+\left|f\left(\frac{r+t-r_{0}}{\varepsilon}\right)\right| \ln \frac{r}{r_{0} \varepsilon} .
$$

Away from the focus, say for $r \geq \delta$, where $\delta>0$ does not depend on $\varepsilon$, we have, from the triangle inequality,

$$
\left|\theta^{\varepsilon}(t, r)-\right| f\left(\frac{t-r-r_{0}}{\varepsilon}\right)\left|\ln \frac{r}{r_{0} \varepsilon}\right| \leq \int_{r_{0}}^{r / \varepsilon} \frac{1}{\sigma}\left|f\left(\frac{t-r-r_{0}}{\varepsilon}+2 \sigma\right)\right| \mathrm{d} \sigma .
$$

The compact support of $f$, along with the assumption $r \geq \delta$, implies that this remainder is bounded, while it is clear that one of the two terms of the left hand side is not. The phase shift can thus be approximated (up to a bounded term) by

$$
\left|f\left(\frac{t-r-r_{0}}{\varepsilon}\right)\right| \ln \frac{r}{r_{0} \varepsilon}+\left|f\left(\frac{r+t-r_{0}}{\varepsilon}\right)\right| \ln \frac{r}{r_{0} \varepsilon} .
$$

The arguments of $|f|$ correspond to the wave propagation: we would consider that the phase shift does not vary like $\ln \varepsilon$ if their factors were opposite, but here they are equal, so we can say that the phase shift is in $-\ln \varepsilon$. It depends on the subsequence $\varepsilon$ chosen to go to zero, and grows arbitrarily large as $\varepsilon$ goes to zero. Now if we define the phase shift as a number belonging to the interval $[0,2 \pi$, that is if we consider the above real modulo $2 \pi$, it seems sensible to call it "random". Even though we consider linear equations, it is clear that this phenomenon is nonlinear, due to the nonlinear coupling.

REMARK 1.5. - Notice that the factor $r_{0}$ in the logarithms in (1.8) is not relevant as far as the phase shift is concerned, for one could write for instance,

$$
\begin{aligned}
\nu_{+}\left(\frac{t-r-r_{0}}{\varepsilon}\right) \mathrm{e}^{i\left|f\left(\frac{t-r-r_{0}}{\varepsilon}\right)\right| \ln \frac{r}{r_{0} \varepsilon}} & =\nu_{+}\left(\frac{t-r-r_{0}}{\varepsilon}\right) e^{i\left|f\left(\frac{t-r-r_{0}}{\varepsilon}\right)\right|\left(\ln \frac{r}{\varepsilon}-\ln r_{0}\right)} \\
& =\widetilde{\nu}_{+}\left(\frac{t-r-r_{0}}{\varepsilon}\right) \mathrm{e}^{i\left|f\left(\frac{t-r-r_{0}}{\varepsilon}\right)\right| \ln \frac{r}{\varepsilon}}
\end{aligned}
$$

where the definition of $\widetilde{\nu}_{+}$is obvious.

In the next section, we reduce the problem to a scattering issue. Section 3 is devoted to existence results and a priori estimates. We deduce the existence of a scattering operator in Section 4 , and finally translate this result to complete the proof of Theorem 1.1, in Section 5.

Acknowledgments. - The idea that such models as (1.2) could lead to random phase shift is due to G. Métivier. The suggestions of J. Rauch and the referee also proved very useful.

TOME $131-2003-\mathrm{N}^{\mathrm{O}} 2$ 


\section{Splitting the variables}

To fix the ideas, we assume that

$$
\operatorname{supp} f, g \subset\left[-z_{0}, z_{0}\right] .
$$

Without changing the nature of the problem, we can assume $r_{0}>z_{0}$.

At time $t=0$, the pulse $v_{-}^{\varepsilon}$ is thus supported in $\left[r_{0}-z_{0} \varepsilon, r_{0}+z_{0} \varepsilon\right]$. Since it propagates at speed minus one (along incoming characteristics), it reaches the origin at time $t=r_{0}-z_{0} \varepsilon$. Therefore it seems sensible to believe that the focusing occurs around $(t, r)=\left(r_{0}, 0\right)$, at scale $\varepsilon$. This is what happens in the case of Eq. (1.1), for $p>2$ (see [6], [4]), and we will see that the same holds in the present. Consequently, we split the variables around the focal point,

$$
\tau=\frac{t-r_{0}}{\varepsilon}, \quad \rho=\frac{r}{\varepsilon} .
$$

Since we are interested in asymptotics in $L^{\infty}$, this splitting has to go with a change of unknown functions that preserves the $L^{\infty}$-norm,

$$
v_{ \pm}^{\varepsilon}(t, r)=\left.\psi_{ \pm}^{r_{0} / \varepsilon}(\tau, \rho)\right|_{\tau=\left(t-r_{0}\right) / \varepsilon, \rho=r / \varepsilon} .
$$

The surprising notation for the index of $\psi$ becomes relevant below. The main point that we want to underscore at this stage is that the functions $\psi_{ \pm}^{r_{0} / \varepsilon}$ do in general depend on $\varepsilon$. The idea is that we expect these functions to converge in some sense as $\varepsilon$ goes to zero. They solve

$$
\left\{\begin{array}{l}
\left(\partial_{\tau} \pm \partial_{\rho}\right) \psi_{ \pm}^{r_{0} / \varepsilon}=\frac{i}{\rho}|f(\tau+\rho)-f(\tau-\rho)|\left(\psi_{-}^{r_{0} / \varepsilon}+\psi_{+}^{r_{0} / \varepsilon}\right), \quad \tau \geq-r_{0} / \varepsilon, \rho>0, \\
\psi_{-}^{r_{0} / \varepsilon}+\psi_{+}^{r_{0} / \varepsilon} \mid \rho=0=0, \\
\left.\psi_{-}^{r_{0} / \varepsilon}\right|_{\tau=-r_{0} / \varepsilon}=\left.g(\tau+\rho) \mathrm{e}^{i|f(\tau+\rho)| \ln r_{0} / \rho}\right|_{\tau=-r_{0} / \varepsilon}, \\
\left.\psi_{+}^{r_{0} / \varepsilon}\right|_{\tau=-r_{0} / \varepsilon}=0 .
\end{array}\right.
$$

The dependence upon $\varepsilon$ has essentially disappeared; it has clearly disappeared from the argument of the logarithm in the initial data (because we initially introduced that strange logarithm in (1.5)), and there will be no more $\varepsilon$ if we just replace $r_{0} / \varepsilon$ by a factor that will eventually go to $+\infty$. We call this factor $n$, even though it may or may not be an integer.

As in [4], we continue these functions to all times by just replacing $\tau=-n$ by $\tau \leq-n$ (in view of the construction of a - modified - Moeller's wave operator). This leads to

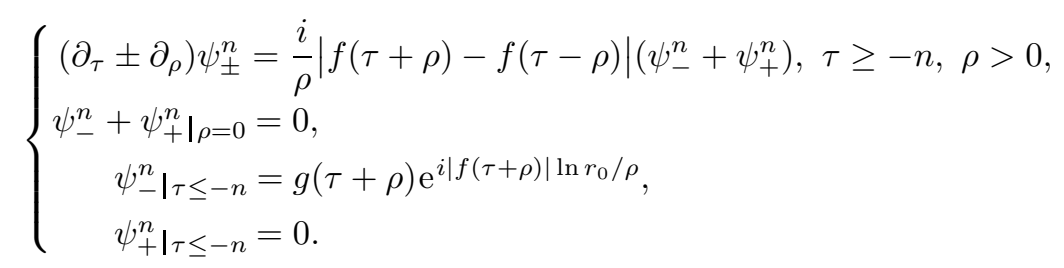

BULletin DE LA SOCIÉtÉ MATHÉMATIQUE DE FRANCE 
It is now time to take advantage of the presence of the factor $i$, which makes the system conservative. The idea is that when $\psi_{-}$and $\psi_{+}$do not interact, their evolution is described by an equation of the form

$$
\left(\partial_{\tau} \pm \partial_{\rho}\right) \psi_{ \pm}^{n}=\frac{i}{\rho}|f(\tau+\rho)-f(\tau-\rho)| \psi_{ \pm}^{n},
$$

that is, an ordinary differential equation along the characteristics (as in [7]). The presence of the factor $i$ makes the solution of such an ordinary differential equation rotate, without altering its amplitude (while if $i$ was replaced by, say, -1 , then the amplitude would decrease to zero, as in [5]). To take this phenomenon into account, we introduce the new change of unknown functions,

$$
\left\{\begin{array}{l}
\widetilde{\psi_{-}^{n}}(\tau, \rho)=\psi_{-}^{n}(\tau, \rho) \exp \left(i \int_{r_{0}}^{\rho} \sigma^{-1}|f(\tau+\rho)-f(\tau+\rho-2 \sigma)| \mathrm{d} \sigma\right) \\
\widetilde{\psi_{+}^{n}}(\tau, \rho)=\psi_{+}^{n}(\tau, \rho) \exp \left(-i \int_{0}^{\rho} \sigma^{-1}|f(\tau-\rho+2 \sigma)-f(\tau-\rho)| \mathrm{d} \sigma\right)
\end{array}\right.
$$

Now, $f$ and $g$ are compactly supported; for $n$ large enough and $\rho>0, \tau \leq-n$, one has, on the support of $g(\rho+\tau)$, and because we assumed $r_{0}>z_{0}$,

$$
\int_{r_{0}}^{\rho} \frac{1}{\sigma}|f(\tau+\rho)-f(\tau+\rho-2 \sigma)| \mathrm{d} \sigma=|f(\tau+\rho)| \ln \frac{\rho}{r_{0}} .
$$

Thus, the pair $\left(\widetilde{\psi_{-}^{n}}, \widetilde{\psi_{+}^{n}}\right)$ solves

$$
\left\{\begin{aligned}
\left(\partial_{\tau} \pm \partial_{\rho}\right) \widetilde{\psi_{ \pm}^{n}} & =\frac{i}{\rho}|f(\tau+\rho)-f(\tau-\rho)| \widetilde{\psi_{\mp}^{n}} \mathrm{e}^{\mp i \phi}, \quad \tau \geq-n, \rho>0 \\
\widetilde{\psi_{-}^{n}}+\widetilde{\psi_{+}^{n}} \mathrm{e}^{i \phi} \mid \rho=0 & =0 \\
\left.\widetilde{\psi_{-}^{n}}\right|_{\tau \leq-n} & =g(\tau+\rho), \\
\left.\widetilde{\psi_{+}^{n}}\right|_{\tau \leq-n} & =0,
\end{aligned}\right.
$$

where

$$
\phi(\tau, \rho)=\int_{0}^{\rho} \frac{1}{\sigma}|f(\tau-\rho+2 \sigma)-f(\tau-\rho)| \mathrm{d} \sigma+\int_{r_{0}}^{\rho} \frac{1}{\sigma}|f(\tau+\rho)-f(\tau+\rho-2 \sigma)| \mathrm{d} \sigma .
$$

Notice that $\phi$ does not depend on $n$ or $\varepsilon$, again because we introduced the right logarithm in the initial data (1.5).

The construction of a wave operator consists in first showing that the functions $\widetilde{\psi_{ \pm}^{n}}$ (hence $\psi_{ \pm}^{n}$ ) converge as $n$ goes to $+\infty$. We first prove some results for fixed $n$.

\section{Results for fixed $n$}

Notation. - For $\psi$ a function of $\tau$ and $\rho$, we will distinguish its $L^{\infty}$-norm in space only, and its $L^{\infty}$-norm in space and time. We will denote

$$
|\psi(\tau)|_{\infty}=\operatorname{ess} \sup _{\rho \in \mathbb{R}_{+}}|\psi(\tau, \rho)|
$$

TOME $131-2003-\mathrm{N}^{\mathrm{O}} 2$ 
and $|\cdot|_{L^{\infty}\left(I \times \mathbb{R}_{+}\right)}$will stand for the usual $L^{\infty}$-norm in space and time.

Using the method of characteristics and the fact that the potential $p$ defined as

$$
p(\tau, \rho)=\frac{1}{\rho}|f(\tau+\rho)-f(\tau-\rho)|,
$$

belongs to $L^{\infty}\left(\mathbb{R} \times \mathbb{R}_{+}\right)$, it is routine to prove local existence and uniqueness of a solution for the problem

$$
\left\{\begin{array}{l}
\left(\partial_{\tau} \pm \partial_{\rho}\right) \varphi_{ \pm}=\frac{i}{\rho}|f(\tau+\rho)-f(\tau-\rho)| \varphi_{\mp} \mathrm{e}^{\mp i \phi}, \quad \tau \geq \tau_{0}, \rho>0 \\
\varphi_{-}+\left.\varphi_{+} \mathrm{e}^{i \phi}\right|_{\rho=0}=0, \\
\varphi_{ \pm \mid \tau=\tau_{0}}=\varphi_{0 \pm},
\end{array}\right.
$$

for any $\tau_{0} \in \mathbb{R}$ and $\varphi_{0 \pm}$ bounded. Global existence for (2.5) is a direct consequence of the following lemma which ensures that the solution $\left(\varphi_{-}, \varphi_{+}\right)$of the above problem is defined for all $\tau \geq \tau_{0}$.

Lemma 3.1. - Let $\varphi_{0 \pm} \in L^{\infty}\left(\mathbb{R}_{+}\right)$and $\tau_{0} \in \mathbb{R}$. There exists a unique solution $\left(\varphi_{-}, \varphi_{+}\right)$of $(3.1)$ in $C\left(\left[\tau_{0}, \infty\right), L^{\infty}\left(\mathbb{R}_{+}\right)\right)$. Moreover, one has, for $\tau \geq \tau_{0}$,

$$
\left|\varphi_{+}(\tau)\right|_{\infty}+\left|\varphi_{-}(\tau)\right|_{\infty} \leq\left(\left|\varphi_{+}\left(\tau_{0}\right)\right|_{\infty}+2\left|\varphi_{-}\left(\tau_{0}\right)\right|_{\infty}\right) \exp \left(2|p|_{L^{\infty}}\left(\tau-\tau_{0}\right)\right) .
$$

Proof. — By Duhamel's rule we have for all $\tau \geq \tau_{0}$,

$$
\varphi_{-}(\tau, \rho)=\varphi_{-}\left(\tau_{0}, \tau+\rho-\tau_{0}\right)+i \int_{\tau_{0}}^{\tau}\left(p \varphi_{+} \mathrm{e}^{i \phi}\right)(\sigma, \rho+\tau-\sigma) \mathrm{d} \sigma
$$

and hence

$$
\left|\varphi_{-}(\tau, \rho)\right| \leq\left|\varphi_{-}\left(\tau_{0}\right)\right|_{\infty}+|p|_{L^{\infty}} \int_{\tau_{0}}^{\tau}\left|\varphi_{+}\right|_{L^{\infty}\left(\left[\tau_{0}, \sigma\right] \times \mathbb{R}_{+}\right)} \mathrm{d} \sigma .
$$

When dealing with $\varphi_{+}$, one must be most careful because of reflections.

- When $\rho-\tau+\tau_{0} \geq 0$, there is no reflection and the same kind of computations as for $\varphi_{-}$yield

$$
\left|\varphi_{+}(\tau, \rho)\right| \leq\left|\varphi_{+}\left(\tau_{0}\right)\right|_{\infty}+|p|_{L^{\infty}} \int_{\tau_{0}}^{\tau}\left|\varphi_{-}\right|_{L^{\infty}\left(\left[\tau_{0}, \sigma\right] \times \mathbb{R}_{+}\right)} \mathrm{d} \sigma .
$$

- When $\rho-\tau+\tau_{0} \leq 0$, there is a reflection, and one has to write

$$
\varphi_{+}(\tau, \rho)=\varphi_{+}(\tau-\rho, 0)+i \int_{\tau-\rho}^{\tau}\left(p \varphi_{-} \mathrm{e}^{-i \phi}\right)(\sigma, \rho-\tau+\sigma) d \sigma .
$$

Thanks to the reflection condition $\varphi_{-}+\varphi_{+} \mathrm{e}^{i \phi} \mid \rho=0=0$ and to (3.2), one obtains

$$
\begin{aligned}
\left|\varphi_{+}(\tau, \rho)\right| \leq\left|\varphi_{-}\left(\tau_{0}\right)\right|_{\infty}+|p|_{L^{\infty}} \int_{\tau_{0}}^{\tau-\rho}\left|\varphi_{+}\right|_{L^{\infty}\left(\left[\tau_{0}, \sigma\right] \times \mathbb{R}_{+}\right)} \mathrm{d} \sigma \\
+|p|_{L^{\infty}} \int_{\tau-\rho}^{\tau}\left|\varphi_{-}\right|_{L^{\infty}\left(\left[\tau_{0}, \sigma\right] \times \mathbb{R}_{+}\right)} \mathrm{d} \sigma .
\end{aligned}
$$

BULLETIN DE LA SOCiÉTÉ MATHÉMATIQUe DE FRANCE 
Inequalities (3.2)-(3.4) yield the general estimate

$$
\begin{aligned}
\left|\varphi_{+}\right|_{L^{\infty}\left(\left[\tau_{0}, \tau\right] \times \mathbb{R}_{+}\right)}+\left|\varphi_{-}\right|_{L^{\infty}\left(\left[\tau_{0}, \tau\right] \times \mathbb{R}_{+}\right)} \leq\left|\varphi_{+}\left(\tau_{0}\right)\right|_{\infty}+2\left|\varphi_{-}\left(\tau_{0}\right)\right|_{\infty} \\
+2|p|_{L^{\infty}} \int_{\tau_{0}}^{\tau}\left(\left|\varphi_{+}\right|_{L^{\infty}\left(\left[\tau_{0}, \sigma\right] \times \mathbb{R}_{+}\right)}+\left|\varphi_{-}\right|_{L^{\infty}\left(\left[\tau_{0}, \sigma\right] \times \mathbb{R}_{+}\right)}\right) \mathrm{d} \sigma
\end{aligned}
$$

from which the result of the lemma follows by Gronwall's Lemma.

In the following proposition, we gather global (and uniform in $n$ ) estimates, and decay properties of the (global) solution $\left(\widetilde{\psi_{-}^{n}}, \widetilde{\psi_{+}^{n}}\right)$ of $(2.5)$.

Proposition 3.2. - The global solution $\left(\widetilde{\psi_{-}^{n}}, \widetilde{\psi_{+}^{n}}\right)$ of $(2.5)$ satisfies the following estimates,

(i) $\left|\widetilde{\psi_{-}^{n}}\right|_{L^{\infty}\left(\mathbb{R} \times \mathbb{R}_{+}\right)}+\left|\widetilde{\psi_{+}^{n}}\right|_{L^{\infty}\left(\mathbb{R} \times \mathbb{R}_{+}\right)} \leq C|g|_{\infty} ;$

(ii) For all $(\tau, \rho) \in \mathbb{R} \times \mathbb{R}_{+}$, one has

$$
\left|\widetilde{\psi_{-}^{n}}(\tau, \rho)\right| \leq \frac{C^{*}}{\langle\tau+\rho\rangle} ;
$$

(iii) For all $(\tau, \rho) \in \mathbb{R} \times \mathbb{R}_{+}$, one has

$$
\left|\widetilde{\psi_{+}^{n}}(\tau, \rho)\right| \leq \frac{C^{*}}{\langle\tau-\rho\rangle} .
$$

The constants $C$ and $C^{*}$ do not depend on $n$.

Proof. - We divide the proof into cases based on the space-time regions that the support of the interaction potential $p$ divides the space-time into. Since the solution propagates freely outside the support of $p$, that support constitutes the "interaction zone" for the solution. We denote it by $I$.

Step 1: Estimates in the interaction zone. - Proving the estimates for $(\tau, \rho)$ belonging to the interaction zone reduces to proving that

$$
\left|\widetilde{\psi_{ \pm}^{n}}(\tau, \rho)\right| \leq C|g|_{\infty}, \quad \text { for all }(\tau, \rho) \in I
$$

and that moreover,

$$
\left|\widetilde{\psi_{\mp}^{n}}(\tau, \rho)\right| \leq \frac{C^{*}}{\langle\rho\rangle}, \quad \text { for all }(\tau, \rho) \in I \text { such that } \pm \tau>0 .
$$

In order to prove (3.5)-(3.6), divide the interaction zone into three subdomains: the bounded triangle $I_{0}:=\left\{(\tau, \rho) ;|\tau| \leq 2 z_{0}-\rho, 0 \leq \rho \leq z_{0}\right\}$, and the unbounded portions below and above the $\rho$-axis, denoted respectively by $I_{-}$ and $I_{+}$(see Fig. 2).

Remark that $\widetilde{\psi_{+}^{n}}$ vanishes on the lower boundary of $I_{-}$, and that one always has

$$
|p| \leq \frac{2\|f\|_{\text {Lip }}}{\langle\rho\rangle}
$$

TOME $131-2003-\mathrm{N}^{\mathrm{O}} 2$ 


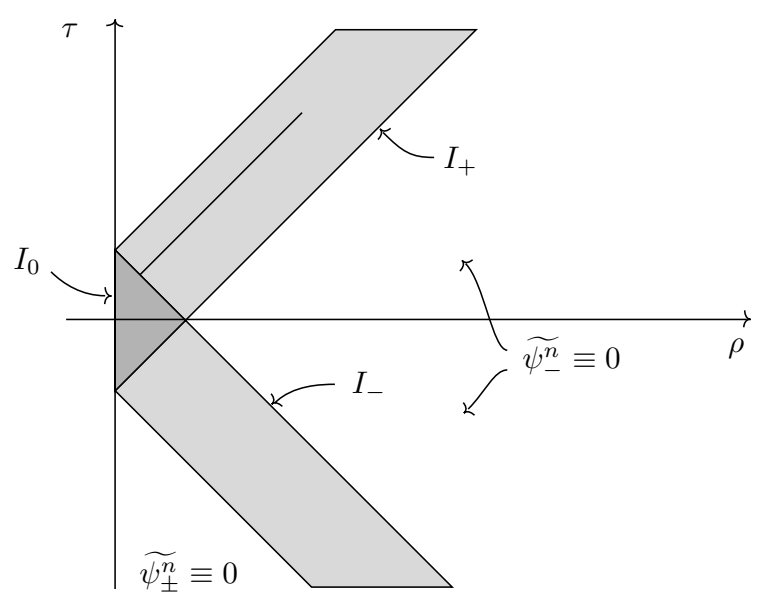

FiguRE 2. Geometry of the propagation.

Since the length of the transverse characteristic crossing $I_{-}$is not greater than $2 z_{0}$, one obtains for all $(\tau, \rho) \in I_{-}$,

$$
\left|\widetilde{\psi_{+}^{n}}(\tau, \rho)\right| \leq \frac{C}{\langle\rho\rangle}\left|\widetilde{\psi_{-}^{n}}\right|_{L^{\infty}\left((-\infty, \tau) \times \mathbb{R}_{+} \cap I_{-}\right)} .
$$

Similar arguments yield for all $(\tau, \rho) \in I_{+}$,

$$
\left|\widetilde{\psi_{-}^{n}}(\tau, \rho)\right| \leq \frac{C}{\langle\rho\rangle}\left|\widetilde{\psi_{+}^{n}}\right|_{L^{\infty}\left((-\infty, \tau) \times \mathbb{R}_{+} \cap I_{+}\right)} .
$$

For any $(\tau, \rho) \in I_{-}$such that $\tau \geq-n$, one has

$$
\widetilde{\psi_{-}^{n}}(\tau, \rho)=g(-n+\rho)+i \int_{-n}^{\tau}\left(p \widetilde{\psi_{+}^{n}} \exp (i \phi)\right)(\sigma, \tau+\rho-\sigma) \mathrm{d} \sigma
$$

which yields, together with (3.7),

$$
\left|\widetilde{\psi_{-}^{n}}(\tau, \rho)\right| \leq|g|_{\infty}+\int_{-n}^{\tau} \frac{C}{\langle\tau+\rho-\sigma\rangle^{2}}\left|\widetilde{\psi_{-}^{n}}\right|_{L^{\infty}\left((-\infty, \sigma) \times \mathbb{R}_{+} \cap I_{-}\right)} \mathrm{d} \sigma .
$$

Since for all $(\tau, \rho) \in I_{-}$, one has $\langle\tau+\rho-\sigma\rangle \geq$ Const $\cdot\langle\sigma\rangle$ for all $\sigma \leq \tau$, the above equation yields

$$
\left|\widetilde{\psi_{-}^{n}}(\tau, \rho)\right| \leq|g|_{\infty}+\int_{-n}^{\tau} \frac{C}{\langle\sigma\rangle^{2}}\left|\widetilde{\psi_{-}^{n}}\right|_{L^{\infty}\left((-\infty, \sigma) \times \mathbb{R}_{+} \cap I_{-}\right)} d \sigma,
$$

from which it is easy to prove $(3.5)$ for $\widetilde{\psi_{-}^{n}}$ and $(\tau, \rho) \in I_{-}$by a Gronwall type argument.

BULLETIN DE LA SOCIÉtÉ MATHÉMATIQUE DE FRANCE 
This result is used together with Lemma 3.1 to prove that (3.5) (and therefore (3.6)) both hold on the bounded triangle $I_{0}$. It remains therefore to prove $(3.5)-(3.6)$ on $I_{+}$.

For all $(\tau, \rho) \in I_{+}, \widetilde{\psi_{+}^{n}}(\tau, \rho)$ is determined by a characteristic line issued from the upper boundary of $I_{0}$ (which does not reflect off the boundary $\rho=0$, see Fig. 2). Since (3.5) holds on $I_{0}$, we know that $\left|\widetilde{\psi_{+}^{n}}(\tau, \rho)\right| \leq C|g|_{\infty}$ on its upper boundary. Using a Gronwall type argument as done for $I_{-}$then yields (3.5) on $I_{+}$.

We have therefore proved (3.5) for the whole interaction zone $I$. The decay estimates (3.6) follow from (3.5) and (3.7)-(3.8).

Step 2: Estimates outside the interaction zone. — Point (i) of the proposition can be deduced immediately from Step 1 , since $\widetilde{\psi_{-}^{n}}$ and $\widetilde{\psi_{+}^{n}}$ propagate freely outside $I$.

Outside the interaction zone, $\widetilde{\psi_{-}^{n}}(\tau, \rho)$ always vanishes, except in the zone above $I_{+}$, where it is determined by free propagation at speed -1 from its values on the upper boundary of $I_{+}$.

Point (ii) of the proposition is therefore a consequence of the previous step of this proof.

Similarly, point (iii) can be deduced from the previous step. The reflection on the boundary $\rho=0$ requires only straightforward modifications.

We finally state a corollary, which is a consequence of the above proof, and will be used in the proof of Th. 1.1, in Sect. 5 .

Corollary 3.3. - Let $\tau_{0} \leq-1$. Then there exists $C$ independent of $\tau_{0}$ and $n>0$ such that,

$$
\sup _{\tau \leq \tau_{0}}\left|\widetilde{\psi_{-}^{n}}(\tau)-g(\tau+.)\right|_{\infty} \leq \frac{C}{\left|\tau_{0}\right|}, \quad \sup _{\tau \leq \tau_{0}}\left|\widetilde{\psi_{+}^{n}}(\tau)\right|_{\infty} \leq \frac{C}{\left|\tau_{0}\right|} .
$$

Proof. — From Eq. (3.9) and Prop. 3.2, (i), one obtains

$$
\left|\widetilde{\psi_{-}^{n}}(\tau, \rho)-g(\tau+\rho)\right| \leq C \int_{-\tau_{0}}^{\infty} \frac{\mathrm{d} \sigma}{\sigma^{2}} .
$$

This is the first estimate of Cor. 3.3. The second estimate is a consequence of Prop. 3.2, (iii).

\section{Construction of the scattering operator}

The key point in the construction of the scattering operator is to prove that the sequence $\left(\widetilde{\psi_{-}^{n}}, \widetilde{\psi_{+}^{n}}\right)$ studied in the previous section converges. This is a consequence of the following proposition.

TOME $131-2003-\mathrm{N}^{\mathrm{O}} 2$ 
Proposition 4.1. - The sequence $\left(\widetilde{\psi_{ \pm}^{n}}\right)_{n}$, where for each $n>0,\left(\widetilde{\psi_{-}^{n}}, \widetilde{\psi_{+}^{n}}\right)$ is the solution of $(2.5)$, converges toward $\widehat{\psi_{ \pm}^{\infty}}$ in $L^{\infty}\left(\mathbb{R}_{\tau} \times \mathbb{R}_{\rho}^{+}\right)$and for all $n>0$,

$$
\left|\widetilde{\psi_{ \pm}^{\infty}}-\widetilde{\psi_{ \pm}^{n}}\right|_{L^{\infty}\left(\mathbb{R}_{\tau} \times \mathbb{R}_{\rho}^{+}\right)}=O\left(\frac{1}{n}\right)
$$

REMARK 4.2. - In our proof, it is essential that the function $\phi$, appearing in (2.5), does not depend on $n$; otherwise, Proposition 3.2 would still hold, but we could not prove the decay estimate (4.2) below. Recall the fact that $\phi$ is independent of $n$ is closely related to the particular form of the initial data (1.5).

Proof. - As in the proof of Prop. 3.2, we first prove the result in the interaction zone $I$, from which the general result can be deduced. In this proof, we take $n_{2} \geq n_{1}>0$.

Step 1: $\left(\widetilde{\psi_{ \pm}^{n}}\right)_{n}$ is a Cauchy sequence on I. - Following the proof of (3.6) it is easy to obtain for all $(\tau, \rho) \in I_{\mp}$

$$
\left|\widetilde{\psi_{ \pm}^{n_{2}}}(\tau, \rho)-\widetilde{\psi_{ \pm}^{n_{1}}}(\tau, \rho)\right| \leq \frac{C}{\langle\rho\rangle}\left|\widetilde{\psi_{\mp}^{n_{2}}}-\widetilde{\psi_{\mp}^{n_{1}}}\right|_{L^{\infty}\left((-\infty, \tau) \times \mathbb{R}_{+} \cap I_{\mp}\right)} .
$$

In order to prove that $\left(\widetilde{\psi_{ \pm}^{n}}\right)_{n}$ is a Cauchy sequence on $I_{-}$, remark that for all $(\tau, \rho) \in I_{-}$such that $\tau \geq-n_{1}$, one can obtain, from (4.2) (as done for (3.9)),

$$
\left|\widetilde{\psi_{-}^{n_{2}}}(\tau, \rho)-\widetilde{\psi_{-}^{n_{1}}}(\tau, \rho)\right| \leq \int_{-n_{2}}^{\tau} \frac{C}{\langle\sigma\rangle^{2}}\left|\widetilde{\psi_{-}^{n_{2}}}-\widetilde{\psi_{-}^{n_{1}}}\right|_{L^{\infty}\left((-\infty, \sigma) \times \mathbb{R}_{+} \cap I_{-}\right)} \mathrm{d} \sigma .
$$

For $\tau \leq-n_{2}$, the left hand side of the above inequality vanishes, while for $-n_{2} \leq \tau \leq-n_{1}$, the same method as above yields

$$
\left|\widetilde{\psi_{-}^{n_{2}}}(\tau, \rho)-\widetilde{\psi_{-}^{n_{1}}}(\tau, \rho)\right| \leq \int_{-n_{2}}^{\tau} \frac{C}{\langle\sigma\rangle^{2}}\left|\widetilde{\psi_{-}^{n_{2}}}\right|_{L^{\infty}\left((-\infty, \sigma) \times \mathbb{R}_{+} \cap I_{-}\right)} \mathrm{d} \sigma .
$$

Since we know by Prop. 3.2 that $\left|\widetilde{\psi_{-}^{n_{2}}}\right|_{L^{\infty}\left(\mathbb{R} \times \mathbb{R}_{+}\right)}$is bounded by a constant independent of $n_{2}$, and since the integral of $1 /\langle\sigma\rangle^{2}$ converges, it follows easily that

$$
\widetilde{\mid \psi_{-}^{n_{2}}}-\left.\widetilde{\psi_{-}^{n_{1}}}\right|_{L^{\infty}\left(\left(-n_{2},-n_{1}\right) \times \mathbb{R}_{+} \cap I_{-}\right)}=O\left(\frac{1}{n_{1}}\right) .
$$

From (4.3)-(4.4), it follows that for all $\tau \leq 0$,

$$
\begin{aligned}
& \left|\widetilde{\psi_{-}^{n_{2}}}-\widetilde{\psi_{-}^{n_{1}}}\right|_{L^{\infty}\left((-\infty, \tau) \times \mathbb{R}_{+} \cap I_{-}\right)} \\
& \quad \leq O\left(\frac{1}{n_{1}}\right)+\int_{-n_{2}}^{\tau} \frac{C}{\langle\sigma\rangle^{2}}\left|\widetilde{\psi_{-}^{n_{2}}}-\widetilde{\psi_{-}^{n_{1}}}\right|_{L^{\infty}\left((-\infty, \sigma) \times \mathbb{R}_{+} \cap I_{-}\right)} \mathrm{d} \sigma,
\end{aligned}
$$

and Gronwall's Lemma proves that $\left(\widetilde{\psi_{-}^{n}}\right)$ is a Cauchy sequence on $I_{-}$and that the estimate (4.1) holds. Thanks to $(4.2)$, this is also the case of $\left(\widetilde{\psi_{+}^{n}}\right)$. Thanks to Lemma 3.1 and to the estimate proved on $I_{-}$(and hence on the lower boundary 
of $\left.I_{0}\right)$, we obtain that $\widetilde{\mid \psi_{ \pm}^{n_{2}}}-\left.\widetilde{\psi_{ \pm}^{n_{1}}}\right|_{L^{\infty}\left(I_{0}\right)}=O\left(1 / n_{1}\right)$. Since this estimate is true on the lowest boundary of $I_{+}$, one obtains for all $(\tau, \rho) \in I_{+}$,

$$
\begin{aligned}
& \left|\widetilde{\psi_{+}^{n_{2}}}-\widetilde{\psi_{+}^{n_{1}}}\right|_{L^{\infty}\left((-\infty, \tau) \times \mathbb{R}_{+} \cap I_{+}\right)} \\
& \quad \leq O\left(\frac{1}{n_{1}}\right)+\int_{0}^{\tau} \frac{C}{\langle\sigma\rangle^{2}}\left|\widetilde{\psi_{+}^{n_{2}}}-\widetilde{\psi_{+}^{n_{1}}}\right|_{L^{\infty}\left((-\infty, \sigma) \times \mathbb{R}_{+} \cap I_{+}\right)} \mathrm{d} \sigma,
\end{aligned}
$$

and Gronwall's Lemma proves the desired result on $I_{+}$for $\left(\widetilde{\psi_{+}^{n}}\right)$. Eq. (4.2) then yields the result for $\left(\widetilde{\psi_{-}^{n}}\right)$, and the proposition is thus proved on $I$.

Step 2. - The result is extended outside the interaction zone as done in the proof of Prop. 3.2.

As a corollary, we obtain,

Corollary 4.3. - There exists a unique $\left(\widetilde{\psi_{-}^{\infty}}, \widetilde{\psi_{+}^{\infty}}\right) \in L^{\infty}\left(\mathbb{R}_{\tau} \times \mathbb{R}_{\rho}^{+}\right)^{2}$ solution of

It satisfies, as $\tau \rightarrow+\infty$,

$$
\left\{\begin{array}{l}
\left(\partial_{\tau} \pm \partial_{\rho}\right) \widetilde{\psi_{ \pm}^{\infty}}=p \widetilde{\psi_{\mp}^{\infty}} \exp (\mp i \phi), \\
\widetilde{\psi_{-}^{\infty}}+\left.\widetilde{\psi_{+}^{\infty}} \exp (i \phi)\right|_{\rho=0}=0, \\
\lim _{\tau \rightarrow-\infty}\left|\widetilde{\psi_{+}^{\infty}}(\tau)\right|_{\infty}=0, \\
\lim _{\tau \rightarrow-\infty}\left|\widetilde{\psi_{-}^{\infty}}(\tau)-g(\tau+\cdot)\right|_{\infty}=0 .
\end{array}\right.
$$

$$
\left|\widetilde{\psi_{-}^{\infty}}(\tau)\right|_{\infty}=O\left(\frac{1}{\tau}\right)
$$

Moreover, there exists $\mu_{+} \in L^{\infty}(\mathbb{R})$ such that $\mu_{+}(\lambda)=O(1 /\langle\lambda\rangle)$ and

$$
\lim _{\substack{\tau \rightarrow \infty \\ \tau-\rho=\lambda}} \widetilde{\psi_{+}^{\infty}}(\tau, \rho)=\mu_{+}(\lambda)
$$

More precisely, for any $(\tau, \rho)$ such that $\tau-\rho=\lambda$ and $\tau \geq 1$, one has

$$
\widetilde{\psi_{+}^{\infty}}(\tau, \rho)-\mu_{+}(\lambda)=O\left(\frac{1}{\tau}\right) \text {. }
$$

Proof. - Of course, $\left(\widetilde{\psi_{-}^{\infty}}, \widetilde{\psi_{+}^{\infty}}\right)$ is the limit of the sequence $\left(\widetilde{\psi_{-}^{n}}, \widetilde{\psi_{+}^{n}}\right)_{n}$, which exists thanks to Prop. 4.1. Estimate (4.5) is a straightforward consequence of Prop. 3.2, (ii). For the behavior of $\widetilde{\psi_{+}^{\infty}}$ when $\tau$ goes to $+\infty$, we distinguish two cases:

- When $|\tau-\rho|>z_{0}$. Then $p(\tau, \rho)$ vanishes for $\tau>z_{0}$ and $\widetilde{\psi_{+}^{\infty}}$ satisfies therefore a free evolution equation. This yields the existence of $\mu_{+}(\lambda)$, and the rate of convergence is trivially satisfied, since $\widetilde{\psi_{+}^{\infty}}(\tau, \rho)=\mu_{+}(\lambda)$ if $\tau>z_{0}$.

- When $|\lambda| \leq z_{0}$ and $\tau-\rho=\lambda$, write

$$
\widetilde{\psi_{+}^{\infty}}(\tau, \rho)=\widetilde{\psi_{+}^{\infty}}\left(z_{0}, z_{0}-\lambda\right)+i \int_{z_{0}-\lambda}^{\rho}\left(\widetilde{\psi_{-}^{\infty}} \exp (-i \phi)\right)(\lambda+\sigma, \sigma) \mathrm{d} \sigma
$$

TOME $131-2003-\mathrm{N}^{\mathrm{O}} 2$ 
and therefore,

$$
\lim _{\substack{\tau \rightarrow \infty \\ \tau-\rho=\lambda}} \widetilde{\psi_{+}^{\infty}}(\tau, \rho)=\widetilde{\psi_{+}^{\infty}}\left(z_{0}, z_{0}-\lambda\right)+i \int_{z_{0}-\lambda}^{\infty}\left(p \widetilde{\psi_{-}^{\infty}} \exp (-i \phi)\right)(\lambda+\sigma, \sigma) \mathrm{d} \sigma,
$$

where the above integral converges thanks to Prop. 3.2, (ii). Defining $\mu_{+}(\lambda)$ as the r.h.s. of the above equation achieves the proof of the fact that $\mu_{+}$is well defined on $\mathbb{R}$. The convergence rate $O(1 / \tau)$ is a consequence of the convergence rate of the above integral.

Finally, for both cases, the decay estimate on $\mu_{+}$is a consequence of Prop. 3.2, (iii).

We can now define the scattering operator.

DeFinition 4.4. - We denote by $S$ the scattering operator

$$
S: g \longmapsto \mu_{+},
$$

with $\mu_{+}$defined as in Prop. 4.3.

REMARK 4.5. - The construction of $S$ is that of a short-range scattering operator, while we claimed in the introduction that it was long-range. In fact, the long-range phenomena are taken into account by substituting $\widetilde{\psi}$ to $\psi$.

REMARK 4.6. - The decay rate $O(1 /\langle\lambda\rangle)$ of $\mu_{+}(\lambda)$ is probably optimal (as in $[6])$. The interaction which occurs for finite times is the reason why the outgoing wave is no longer compactly supported.

\section{Back to the pulses}

We can now complete the proof of Th. 1.1. From (2.2) and (2.4), we have

$$
\left\{\begin{array}{l}
v_{-}^{\varepsilon}(t, r)=\left.\widetilde{\psi_{-}^{r_{0} / \varepsilon}}(\tau, \rho) \mathrm{e}^{-i \int_{r_{0}}^{\rho} \sigma^{-1}|f(\tau+\rho)-f(\tau+\rho-2 \sigma)| \mathrm{d} \sigma}\right|_{\tau=\frac{t-r_{0}}{\varepsilon}, \rho=\frac{r}{\varepsilon}}, \\
v_{+}^{\varepsilon}(t, r)=\left.\widetilde{\psi_{+}^{r_{0} / \varepsilon}}(\tau, \rho) \mathrm{e}^{i \int_{0}^{\rho} \sigma^{-1}|f(\tau-\rho+2 \sigma)-f(\tau-\rho)| \mathrm{d} \sigma}\right|_{\mid \tau=\frac{t-r_{0}}{\varepsilon}, \rho=\frac{r}{\varepsilon}} .
\end{array}\right.
$$

Therefore, Prop. 3.2 clearly implies the existence part of Th. 1.1.

Let $0 \leq t \leq r_{0}-z_{0} \varepsilon$. Cor. 3.3 implies that, in $L_{r}^{\infty}$,

$$
v_{-}^{\varepsilon}(t, r)=\left.g(\tau+\rho) \mathrm{e}^{-i \int_{r_{0}}^{\rho} \sigma^{-1}|f(\tau+\rho)-f(\tau+\rho-2 \sigma)| \mathrm{d} \sigma}\right|_{\mid \tau=\frac{t-r_{0}}{\varepsilon}, \rho=\frac{r}{\varepsilon}}+O\left(\frac{\varepsilon}{r_{0}-t}\right) .
$$

To complete the proof of the asymptotics for $v_{-}^{\varepsilon}$ before focusing, we use the following

LEMMA 5.1. - On the support of the amplitude $g(\tau+\rho)_{\mid \tau=\frac{t-r_{0}}{\varepsilon}, \rho=\frac{r}{\varepsilon}}$, we have the equality

$$
\int_{r_{0}}^{r / \varepsilon} \frac{1}{\sigma}\left|f\left(\frac{r+t-r_{0}}{\varepsilon}\right)-f\left(\frac{r+t-r_{0}}{\varepsilon}-2 \sigma\right)\right| \mathrm{d} \sigma=\left|f\left(\frac{r+t-r_{0}}{\varepsilon}\right)\right| \ln \frac{r}{r_{0} \varepsilon} .
$$

BULLETIN DE LA SOCiÉtÉ MATHÉMATIQUe DE FRANCE 
Proof. - The amplitude is supported in $-z_{0} \varepsilon \leq r+t-r_{0} \leq z_{0} \varepsilon$. We want to prove that when $\sigma$ is between $r_{0}$ and $r / \varepsilon$,

We distinguish two cases.

$$
f\left(\frac{r+t-r_{0}}{\varepsilon}-2 \sigma\right) \equiv 0 .
$$

First case. - If $r_{0} \leq r / \varepsilon$ then the maximum value for $\left(r+t-r_{0}\right) / \varepsilon-2 \sigma$ is when $\sigma=r_{0}$, and it is

$$
\frac{r+t-r_{0}}{\varepsilon}-2 r_{0} \leq z_{0}-2 r_{0}
$$

because of the support of $g$. We assumed $r_{0}>z_{0}$, therefore the above term is less than $-z_{0}$. This proves the lemma in this case, because of the compact support of $f$.

Second case. - If $r_{0} \geq r / \varepsilon$ then the maximum value for $\left(r+t-r_{0}\right) / \varepsilon-2 \sigma$ is when $\sigma=r / \varepsilon$. Since we assumed $t \leq r_{0}-z_{0} \varepsilon$, it is

$$
\frac{t-r-r_{0}}{\varepsilon} \leq \frac{r_{0}-2 z_{0} \varepsilon-r-r_{0}}{\varepsilon} \leq-z_{0},
$$

and we conclude as in the first case.

This implies the estimate, for $0 \leq t \leq r_{0}-z_{0} \varepsilon$,

$$
\left\|v_{-}^{\varepsilon}(t, r)-g\left(\frac{r+t-r_{0}}{\varepsilon}\right) \mathrm{e}^{i\left|f\left(\left(r+t-r_{0}\right) / \varepsilon\right)\right| \ln r_{0} \varepsilon / r}\right\|_{L_{r}^{\infty}}=O\left(\frac{\varepsilon}{r_{0}-t}\right) .
$$

The estimate

$$
\left\|v_{+}^{\varepsilon}(t, r)\right\|_{L_{r}^{\infty}}=O\left(\frac{\varepsilon}{r_{0}-t}\right)
$$

is a straightforward consequence of (5.1) and the second part of Cor. 3.3. This completes the proof of the asymptotics before focusing (the asymptotics for $r_{0}-t=O(\varepsilon)$ gives no information, for it just says that the difference between two bounded functions is bounded - not necessarily small).

For the asymptotics after the focusing time, one has immediately, from Prop. 3.2, (ii) and (5.1),

$$
\left\|v_{-}^{\varepsilon}(t, r)\right\|_{L_{r}^{\infty}}=O\left(\frac{\varepsilon}{t-r_{0}}\right), \quad t \geq r_{0}+\varepsilon .
$$

Let $t \geq r_{0}+\varepsilon$. From (5.1) and (4.1),

$$
v_{+}^{\varepsilon}(t, r)=\left.\widetilde{\psi_{+}^{\infty}}(\tau, \rho) \mathrm{e}^{i \int_{0}^{\rho} \sigma^{-1}|f(\tau-\rho+2 \sigma)-f(\tau-\rho)| \mathrm{d} \sigma}\right|_{\tau=\frac{t-r_{0}}{\varepsilon}, \rho=\frac{r}{\varepsilon}}+O(\varepsilon) .
$$

From (4.6), we deduce

$v_{+}^{\varepsilon}(t, r)=\left.\mu_{+}(\tau-\rho) \mathrm{e}^{i \int_{0}^{\rho} \sigma^{-1}|f(\tau-\rho+2 \sigma)-f(\tau-\rho)| \mathrm{d} \sigma}\right|_{\tau=\frac{t-r_{0}}{\varepsilon}, \rho=\frac{r}{\varepsilon}}+O\left(\varepsilon+\frac{\varepsilon}{t-r_{0}}\right)$.

Define $\nu_{+}$as

$$
\nu_{+}(\lambda)=\mu_{+}(\lambda) \exp \left(i \int_{0}^{r_{0}} \frac{1}{\sigma}|f(\lambda+2 \sigma)-f(\lambda)| \mathrm{d} \sigma\right) .
$$

TOME $131-2003-\mathrm{N}^{\mathrm{O}} 2$ 
From Cor. 4.3, $\nu_{+}$is bounded and has algebraic decay

$$
\nu_{+}(\lambda)=O\left(\frac{1}{\langle\lambda\rangle}\right)
$$

We clearly have

$$
\begin{aligned}
v_{+}^{\varepsilon}(t, r) & =\nu_{+}(\tau-\rho) \mathrm{e}^{i \int_{r_{0}}^{\rho} \sigma^{-1}|f(\tau-\rho+2 \sigma)-f(\tau-\rho)| \mathrm{d} \sigma} \mid \begin{array}{l}
\tau=\frac{t-r_{0}}{\varepsilon}, \rho=\frac{r}{\varepsilon} \\
\end{array} \quad O\left(\varepsilon+\frac{\varepsilon}{t-r_{0}}\right) \\
& =\nu_{+}\left(\frac{t-r-r_{0}}{\varepsilon}\right) \mathrm{e}^{i \theta^{\varepsilon}(t, r)}+O\left(\varepsilon+\frac{\varepsilon}{t-r_{0}}\right),
\end{aligned}
$$

where $\theta^{\varepsilon}$ is defined in Th. 1.1, and can be approximated by

$$
\theta^{\varepsilon}(t, r) \underset{\varepsilon \rightarrow 0}{\sim}\left|f\left(\frac{t-r-r_{0}}{\varepsilon}\right)\right| \ln \frac{r}{r_{0} \varepsilon},
$$

at least for $r \gg \varepsilon$, as mentioned in Remarks 1.4 and 1.5. Since $\nu_{+}$has algebraic decay, it is (possibly) larger than the remainder term only when $t-r-r_{0}=O(\varepsilon)$. In this neighborhood of the line $t-r-r_{0}=0$, we will have $r \gg \varepsilon$ provided that $t-r_{0} \gg \varepsilon$, that is, when the wave has left the focal point. This completes the proof of the first two points of Th. 1.1.

The last point follows from (5.1) and Proposition 4.1, by defining

$$
\left\{\begin{array}{l}
\mathrm{V}_{-}(\tau, \rho)=\widetilde{\psi_{-}^{\infty}}(\tau, \rho) \mathrm{e}^{-i \int_{r_{0}}^{\rho} \sigma^{-1}|f(\tau+\rho)-f(\tau+\rho-2 \sigma)| \mathrm{d} \sigma} \\
\mathrm{V}_{+}(\tau, \rho)=\widetilde{\psi_{+}^{\infty}}(\tau, \rho) \mathrm{e}^{i \int_{0}^{\rho} \sigma^{-1}|f(\tau-\rho+2 \sigma)-f(\tau-\rho)| \mathrm{d} \sigma}
\end{array}\right.
$$

As noticed in Remark 1.5, the introduction of the value $r_{0}$ to split the previous integral into two parts is arbitrary. Nevertheless, replacing $r_{0}$ by any other positive number keeps the phase shift written in Remark 1.5, plus constant times $|f|$. That term can be considered as part of the amplitude (see Remark 1.5), which leaves the definition of the phase shift unchanged.

\section{BIBLIOGRAPHY}

[1] Alterman (D.) \& Rauch (J.) - Nonlinear geometric optics for short pulses, J. Differential Equations, t. 178 (2002), no. 2, pp. 437-465.

[2] CARLES (R.) - Geometric optics with caustic crossing for some nonlinear Schrödinger equations, Indiana Univ. Math. J., t. 49 (2000), no. 2, pp. 475551.

[3] _ Geometric optics and long range scattering for one-dimensional nonlinear Schrödinger equations, Comm. Math. Phys., t. 220 (2001), no. 1, pp. $41-67$.

[4] Carles (R.) \& Rauch (J.) - Focusing of Spherical Nonlinear Pulses in $\mathbb{R}^{1+3}$, II. Nonlinear Caustic, to appear in Rev. Mat. Iberoamericana. 
[5] - Absorption d'impulsions non linéaires radiales focalisantes dans $\mathbb{R}^{1+3}$, C. R. Acad. Sci. Paris Sér. I Math., t. 332 (2001), no. 11, pp. 985990.

[6] _ Diffusion d'impulsions non linéaires radiales focalisantes dans $\mathbb{R}^{1+3}$, C. R. Acad. Sci. Paris Sér. I Math., t. 332 (2001), no. 12, pp. $1077-$ 1082 .

[7] $\_$Focusing of spherical nonlinear pulses in $\mathbb{R}^{1+3}$, Proc. Amer. Math. Soc., t. 130 (2002), no. 3, pp. 791-804.

[8] _ Focusing of Spherical Nonlinear Pulses in $\mathbb{R}^{1+3}$, III. Sub and Supercritical cases, Preprint, 2002.

[9] DuistermaAt (J.J.) - Oscillatory integrals, Lagrange immersions and unfolding of singularities, Comm. Pure Appl. Math., t. 27 (1974), pp. $207-$ 281.

[10] Hunter (J.) \& Keller (J.) - Caustics of nonlinear waves, Wave motion, t. 9 (1987), pp. 429-443.

[11] Joly (J.-L.), MÉtivier (G.) \& RAuch (J.) - Focusing at a point and absorption of nonlinear oscillations, Trans. Amer. Math. Soc., t. 347 (1995), no. 10, pp. 3921-3969.

[12] - Several recent results in nonlinear geometric optics, in Partial differential equations and mathematical physics (Copenhagen, 1995; Lund, 1995), Birkhäuser Boston, Boston, MA, 1996, pp. 181-206.

[13] Caustics for dissipative semilinear oscillations, Mem. Amer. Math. Soc., vol. 144, no. 685, American Mathematical Society, Providence, 2000.

[14] LAx (P.D.) - Asymptotic solutions of oscillatory initial value problems, Duke Math. J., t. 24 (1957), pp. 627-646.

[15] Ludwig (D.) - Uniform asymptotic expansions at a caustic, Comm. Pure Appl. Math., t. 19 (1966), pp. 215-250. 